\title{
THE CLINICAL FEATURES AND RESPONSE TO CORTISONE OF MENOPAUSAL MUSCULAR DYSTROPHY
}

\author{
BY
}

\section{G. MILTON SHY and DONALD McEACHERN}

From the Department of Neurology and Neurosurgery, McGill University, and the Montreal Neurological Institute, Montreal

We describe here a form of myopathy which occurs predominantly in women during the climacteric period or after, and appears to have characteristic features. The condition has been largely overlooked in the past. Its recognition is of special importance since improvement may occur during treatment with wheat germ oil or cortisone.

Our clinical experience is based upon a study of 12 patients, of whom 11 were women and one was a man. Six of the women were treated with wheat germ oil, and four showed notable improvement. The details are described in the preceding paper. Five patients, four women (two of them from the wheat germ oil series) and one man, have been treated with cortisone, with dramatic improvement in all instances. Details of the trial of cortisone in various neuromuscular disorders have been described in a previous paper (Shy, Brendler, Rabinovitch, and McEachern, 1950).

In this report we present the clinical features of this condition, together with pathological findings obtained by muscle biopsies. The use of maintenance doses of cortisone is also discussed.

Little reference to this condition can be found in the literature. KNevin (1936) described two cases of late dystrophy which were undoubtedly of this type. He also reviewed the literature. Other probable cases are noted in the communication by Bramwell (1922). The cases of late myopathy described by Barnes (1932) may be different! 'They occurred in families in which other members showed myopathic disorders at an early age."

\section{Clinical Features}

The typical picture is that of progressive weakness (sometimes rapid, sometimes slow) of the hip and shoulder girdle muscles. This leads to difficulty in ascending stairs or in rising from a chair without help from the arms. There is trouble, too, in raising the arms above the head, as in combing the hair, or in reaching up to high shelves. It progresses to the point where a waddling gait is evident, and the patient can no longer climb stairs. Unpredictable falls are common, and this symptom may precede all others by months or years. There is no warning and the legs just suddenly give way, although the patient may still be able to walk for many blocks on the level at this stage. There is no complaint of facial weakness, difficulty in swallowing or double vision, and power in the hands and feet remains normal.

Examination of the individual muscles reveals profound weakness of the iliopsoas muscles, the quadriceps femoris groups, the glutei, and the adductors of the lower extremities. In the upper extremities the supraspinati, the infraspinati, the deltoids, serratus magnus, triceps, and biceps are usually the most affected. On the whole, the lower extremities are more severely affected than the upper, but this may be a reflection of the greater weight bearing upon these structures. The condition may be so severe in the later stages that the affected muscles are not only powerless against resistảnce but against gravity alone. All of our patients have been examined by the method described in the monograph on peripheral nerve injury published by the Medical Research Council (1942).

Palpation reveals a "soft" consistency in the affected muscles. Visible wasting is unusual, perhaps because the patients are often well nourished, but occasionally the quadriceps femoris groups are seen to be atrophied.

Deep reflexes may be depressed in the muscles involved in proportion to the severity of involvement. Most of the muscles contributing to the usual reflexes are relatively unaffected, however, and the usual reflexes are therefore often lively. No signs of central nervous system involvement have been elicited. The thyroid is not enlarged, and the basal 
metabolic rate is normal, in contrast to the low rates observed in juvenile dystrophy and dystrophia myotonica. Nevertheless a few of our patients have given a history of hyperthyroidism or of thyroidectomy in preceding years.

A gross increase in the ratio of creatine to the combined creatine and creatinine urinary output, as observed almost constantly in juvenile pseudohypertrophic dystrophy, is not a marked feature in these cases. Creatinuria is surprisingly small.

Other pathological disorders, gout and proliferative arthritis, were observed in two patients. The musculature affected, however, was not related to the affected joints.

\section{Pathology}

The picture is that of a dystrophy. We differentiate a dystrophic process from atrophy secondary to nerve degeneration by the following points : denervated muscle is affected in groups of fibres probably subserved by " motor units"; dystrophic muscle, on the other hand, may show but a single abnormal fibre surrounded by histologically normal tissue. Necrosis with phagocytosis occurs in the more acute dystrophies, and cross striations are lost relatively early, whereas in neural atrophy striations may be present until late in the process. A large amount of fatty infiltration and replacement may eventually occur in dystrophic muscle, but not so commonly in the atrophic muscle. Hyalin changes, as seen by chromatic staining, have not been reliable indices of muscular dysfunction and they may indicate difficulty in fixation technique. Silver staining of nerve endings is of use in early cases. The muscle spindles, the interfusal fibres, are apparently spared in both dystrophies and neural atrophies.

At biopsy the muscle in the cases of menopausal dystrophy is usually pale. Biopsy has always been performed on the muscle most available and most affected clinically. Some muscles, e.g., iliopsoas, cannot be biopsied, but the nearest affected ones, e.g., quadriceps femoris, have then been chosen.

On microscopical examination* the main abnormality is patchy degeneration or necrosis of individual fibres, usually accompanied by phagocytosis of these portions. This process may affect but part of a muscle fibre, and the rest of it may be normal (Fig. 1). These areas are quite numerous and one

\footnotetext{
* Sections of muscle removed were allowed to relax in isotonic saline for 20 minutes, and then placed in acidified zenkers. They were placed at a slight tension along the longitudinal axis by a weight tied to the distal end. Where possible both longitudinal and cross sections were cut.

Cross striations were studied by a light Mallory phosphotungstic
and cross sections were cut. acid stain, since it is believed that the myofibril and its disc are probably impregnated with this stain. Other sections were stained with haematoxylin and eosin, and haematoxylin and Van Gieson stain.
}

need not search long to find them. Sometimes two such areas may be present in one low-power field (Fig. 2). There is usually proliferation of the sarcolemmal nuclei, and in some instances the only abnormality may be a central migration of the swollen sarcolemmal nuclei into the fibre. In advanced cases connective tissue elements and fat are predominant (Fig. 3). Aside from central migration of the nuclei, loss in cross striation is the earliest sign of muscle involvement.

When the dystrophy is of long standing, secondary changes are almost a constant finding. This is reflected in thickening of the media of the arterial walls. Occasionally some interstitial cellular response has been noted, but this is unusual as contrasted to states of dermatomyositis*.

Malamud, Nelson, and Evans (1949), Pappenheimer (1943a, 1943b) and others have described the pathology of vitamin E deficiency in Rodentia. This is a wide-spread dystrophic process with multiple areas of degeneration and necrosis. It is different from the dystrophies of human childhood, where necrosis is rare. Sections from patients with menopausal dystrophy, however, resemble those from the vitamin E-deficient animal. Necrosis is a common factor and it is relatively widespread in the involved muscles. Active phagocytosis is a characteristic of each, making such areas relatively easy to find on rapid scanning of the sections.

\section{Cortisone : Laboratory Studies and Therapeutic Results}

Cortisone was administered to five patients by the intramuscular route in a standard suspension of the acetate $(25 \mathrm{mg}$. per $\mathrm{ml}$.). No more than $50 \mathrm{mg}$. was administered in each depot. The first day of administration, $200 \mathrm{mg}$., or four depots, were used. Each successive day two depots $(100 \mathrm{mg}$.) were administered, for an average duration of 21 days. The fall of circulating eosinophils in the blood was determined at daily and then at three-day intervals. Glucose tolerance curves were made at five-day intervals. The total urinary creatine and creatinine excretion was determined daily. "The patients were all on a meat-free diet. Electromyograms were obtained in all five cases. Because of reports of electroencephalographic changes under ACTH therapy (Hoefer and Glaser, 1950), an initial electroencephalogram was obtained in each instance, and also one at the height of therapy. The muscle power of each patient was graded every two days. Where possible, muscle power was also tested by weight-pulley mechanisms, quantitative stair climbing, and ability to rise out of chairs of graded

\footnotetext{
* In extreme cases one cannot distinguish menopausal dystrophy from other forms of advanced dystrophy.
} 
FIG. $1(\times 240)$. - This demonstrates the breaking up of a portion of several fibres in an otherwise normal field. The cross-striations can be traced from the top of the section to the initial point of necrosis. Note aggregation of sarcoplasm and apparent phagocytic response.

FIG. 2 ( $\times 550)$.-Focal necrosis is by no means rare. Here two fibres are affected in a single high-power field.

FIG. $3(\times 240)$.-Advanced dystrophy with fat and connective tissue replacement. At this stage the process cannot be distinguished from other types of muscle dystrophy.
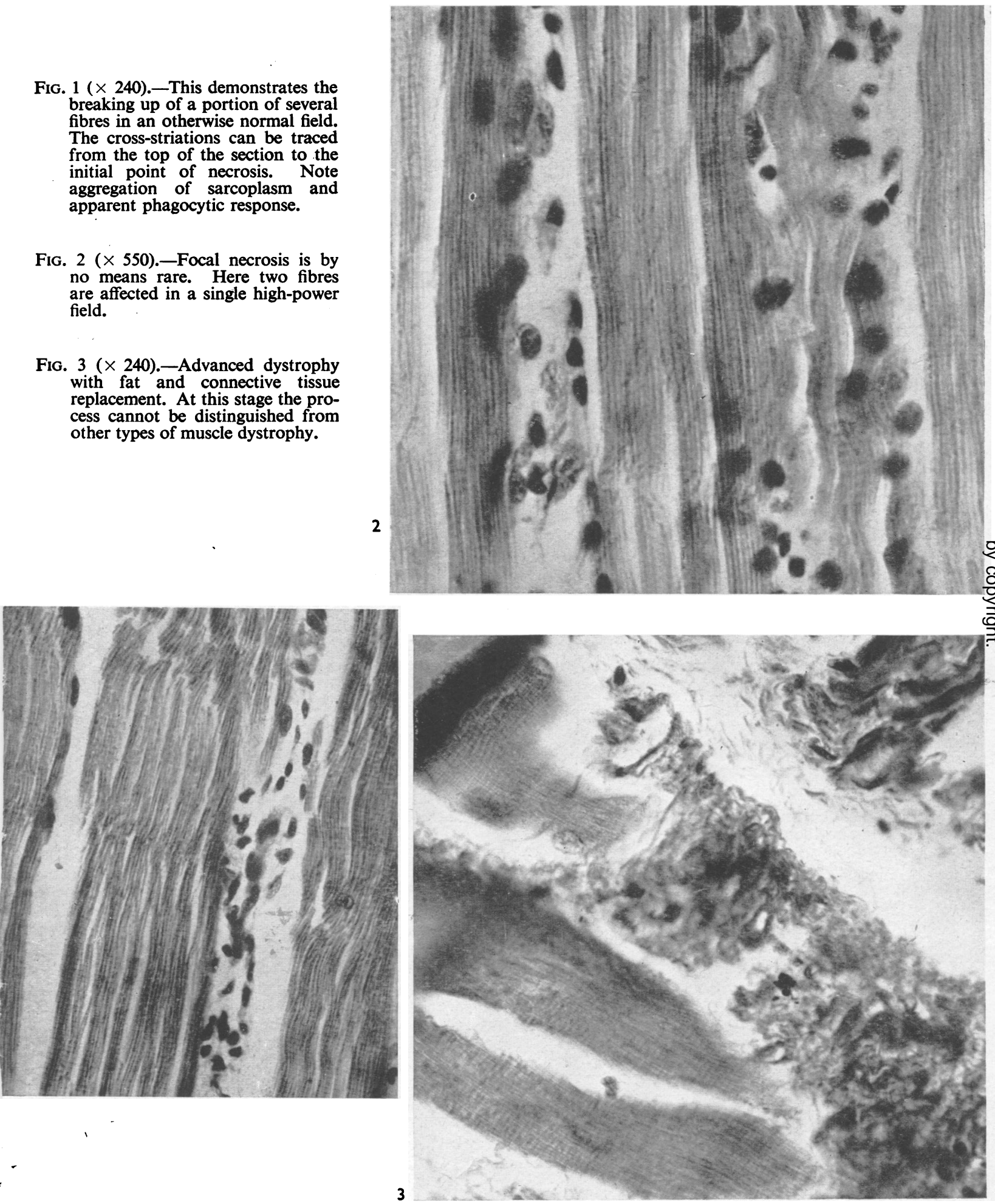

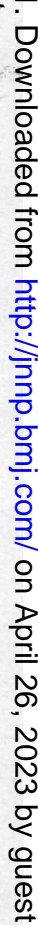


height. Patients were weighed daily, and blood pressure readings obtained twice daily.

By the fourth to sixth day of treatment all of these patients began to show marked improvement. The patient R.P. (Case 1), whose biopsy section is shown in Fig. 4, could, before treatment, ascend only three steps in 20 seconds by pulling herself with her arms. After two weeks she could mount 40 steps in 20 seconds without the use of her arms. All patients had a general euphoria and feeling of well-being. Sleeplessness was common, but not of the unpleasant kind. The experiment was terminated by substituting for the cortisone a suspension of cholesterol which resembled the cortisone in every detail. All patients began to relapse in about six days.

A marked fall in circulating eosinophils occurred in all cases. No marked change in the urinary creatine output was found. Glucose tolerance curves in all patients rose to high normal levels. Weight increased along with fluid retention, and was promptly lost by diuresis on lower dosages of cortisone. No toxic manifestations, such as hypertension, hirsutism, moon face, occurred. No change in the electoencephalogram was found in any of the five cases: Wound (biopsy) healing was delayed in some cases until the time of cholesterol control, and old operative and traumatic scars seemed to soften in texture.

\section{Maintenance Therapy with Cortisone}

For the past six months we have been faced with the problem of maintenance therapy : 100 to $150 \mathrm{mg}$. every two days approximates the optimum dosage in these cases. We have two patients on less medication and one on trore than this amount. These patients are as dependent ùpon cortisone as are diabetics upon insulin. The prospect of a preparation which can be taken by mouth is very cheering to them.

The problem of fluid accumulation on the larger dosages becomes a real one. The patient receiving the most cortisone ( $200 \mathrm{mg}$. every two days) gained about $2 \mathrm{lb}$. weekly from excess fluid. We have recently placed her upon a low sodium diet with a fluid loss of $2 \mathrm{lb}$. weekly. To date we have had only two patients with this difficulty. It is recommended that the blood pressure, weight, and blood and urinary sugars be followed on all patients on maintenance therapy, at least for a time.

\section{Case Reports}

Case 1 (R. P., female, aged 52). - This patient was admitted with complaints of progressive weakness of the lower limbs of three years' duration. The onset was shortly after the menopause. This was accompanied by unpredictable falls, and by mild occipital and upper cervical aching.

No muscular atrophy was seen but the affected muscles felt soft. The chief muscular weakness lay in the quadriceps, iliopsoas, and the gluteus maximus muscles. Knee jerks were absent but achilles jerks were present. Reflexes in the upper extremities were quite brisk.

There was little abnormality in creatine output, the figures on three consecutive days before cortisone therapy being as follows :-

\begin{tabular}{c|c|c|c}
\hline $\begin{array}{c}\text { Urine } \\
\text { Volume } \\
(\mathrm{ml} .)\end{array}$ & $\begin{array}{c}\text { Specific } \\
\text { Gravity }\end{array}$ & $\begin{array}{c}\text { Creatinine } \\
\text { (g./24 hrs. })\end{array}$ & $\begin{array}{c}\text { Creatine } \\
(\mathrm{g} . / 24 \mathrm{hrs} .)\end{array}$ \\
\hline 1155 & 1.015 & 0.50 & 0.12 \\
2050 & 1.012 & 0.62 & 0.11 \\
1400 & 1.011 & 0.56 & 0.10 \\
\hline
\end{tabular}

The glucose tolerance curve was within normal limits. Serum sodium was $320 \mathrm{mg} . \%$, potassium $18.3 \mathrm{mg}$. \%, chlorides $356 \mathrm{mg} . \%$, and the carbon dioxide combining power was 43 volumes \%.

This patient was placed on the usual cortisone regime. There was dramatic improvement as described above. Her weight increased on heavier dosage of cortisone and it had to be dropped for a period to $50 \mathrm{mg}$. daily. There was a sharp fall in the circulating eosinophils. No change could be noted in the serum potassium or sodium levels. The blood pressure did not rise appreci- $\theta$ ably. The muscle picture can be seen in Fig. 3. This shows multiple areas of isolated necrosis with loss of cross striations. There is also clumping of the sarcoplasm and disappearance of the sarcolemmal sheaths, and isolated atrophic degenerated fibres. There is a minimal interstitial histiocytic response.

Case 2 (B. M., aged 59).-Ten years ago this patient noted rapidly progressing weakness of the hip muscles in climbing stairs and rising from chairs. Symptoms began six months after the menopause, and were accompanied by episodes of unpredictable falls. She was placed upon wheat germ oil two years after the onset of symptoms, with marked improvement, maintained for three years, followed by recurrence of symptoms. In 1947 periodic joint pains led to investigation and to the diagnosis of mild gout. Upon admission, the patient could no longer mount or descend steps without support, and she walked only with a cane.

Examination showed maximal weakness in the glutei, the iliopsoas, and the quadriceps muscles. The deltoid, supraspinati, and infraspinati showed some weakness as well. Distal muscle power was well preserved. She thad a more marked creatinuria than the first patient ciled, but still not equal to that in the juvenile dystrophies. For two days without treatment the creatine values were:-

\begin{tabular}{c|c|c|c}
\hline $\begin{array}{c}\text { Volume } \\
(\mathrm{ml} .)\end{array}$ & $\begin{array}{c}\text { Specific } \\
\text { Gravity }\end{array}$ & $\begin{array}{c}\text { Creatinine } \\
(\mathrm{g} . / 24 \mathrm{hrs} .)\end{array}$ & $\begin{array}{c}\text { Creatine } \\
(\mathrm{g} . / 24 \mathrm{hrs})\end{array}$ \\
\hline 1100 & 1.018 & $\mathbf{0 . 6 4 4}$ & 0.332 \\
1360 & 1.018 & 0.608 & 0.254 \\
\hline
\end{tabular}



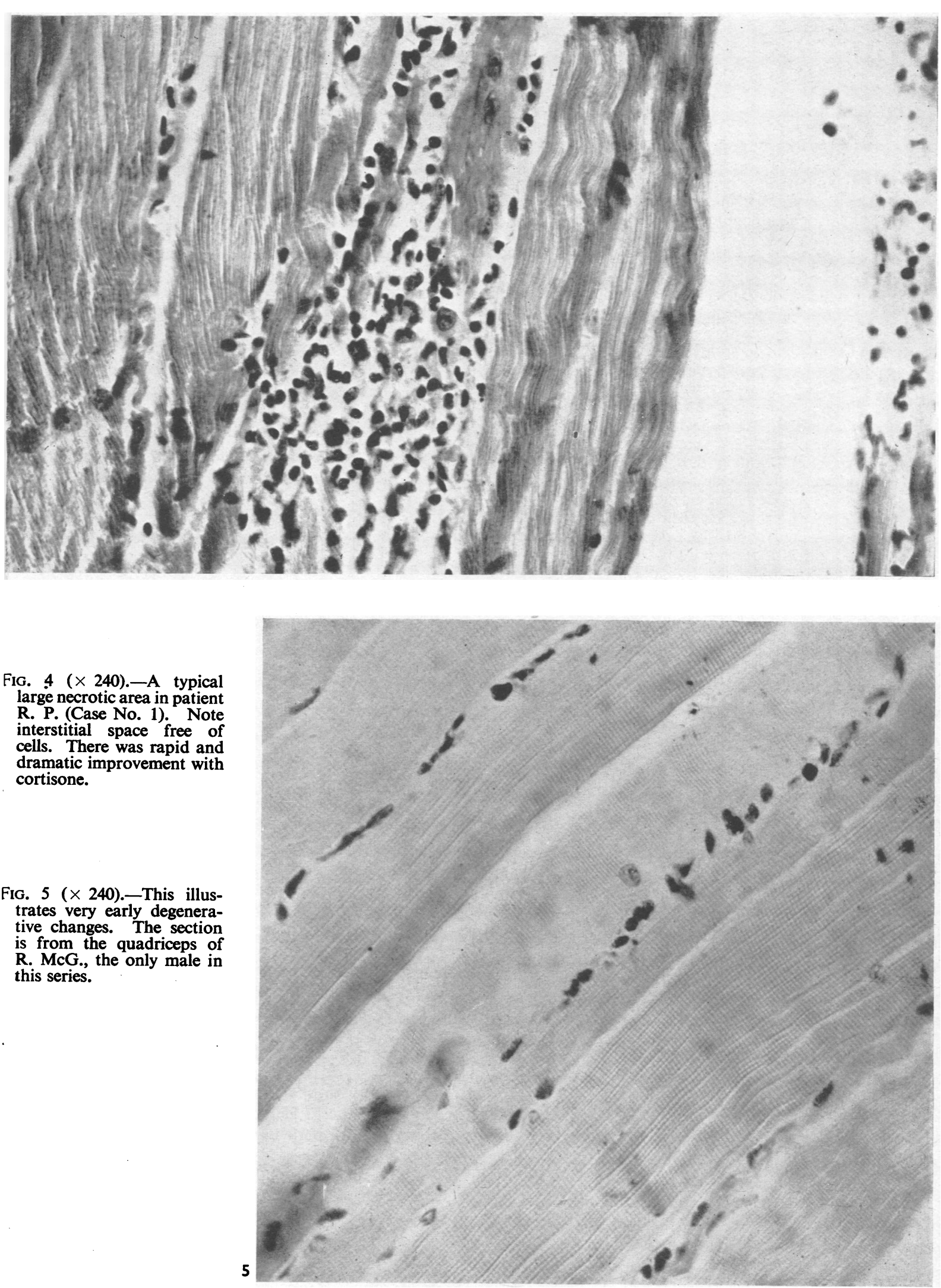

Fig. $4(\times 240) .-A$ typical large necrotic area in patient R. P. (Case No. 1). Note interstitial space free of cells. There was rapid and dramatic improvement with cortisone.

Fig. $5(\times 240)$. -This illustrates very early degenerative changes. The section is from the quadriceps of R. McG., the only male in this series. 
A rapid increase of muscle power was noted by the fifth therapeutic day. The pains associated with the gout also markedly improved. Unlike other cases, this patient has required large maintenance doses of cortisone, up to $200 \mathrm{mg}$. every other day, to maintain improvement. Due to exogenous fluid accumulation we have recently placed her upon ammonium chloride too.

Case 3 (R. McG., aged 58).- - This patient, the only man in our series, gave a history of a rapid onset of weakness of proximal girdle musculature beginning nine months before. This involved the upper extremities, so that he had difficulty in dressing himself. He walked with a cane, could not rise from a chair unassisted, and had great difficulty in getting out of bed.

Examination showed some wasting of the calves. The iliopsoas group was so weak that he could not raise his extended leg from the bed more than five times. The greatest weakness again was in the iliopsoas, quadriceps, and glutei. The quadriceps were possibly more affected than in the other cases.

Biopsy suggested the diagnosis of early dystrophy with early necrotic degeneration. These changes were not marked, and in the estimation of most observers deviated but little from normal (Fig. 5).

This patient responded dramatically to cortisone, and at the time of discharge he could raise either extended leg from the bed 30 times without fatigue. He could also rise from a chair without assistance and could walk without a cane. He is holding improvement on $200 \mathrm{mg}$. of cortisone a week - the smallest maintenance dosage we have been able to use so far.

Case 4 (S. R., aged 68). - This elderly lady noticed isolated episodes of unpredictable falling some four or five years before admission. This was followed within a year by difficulty in climbing stairs and getting out of chairs and in combing her hair. More recently, over the-" past nine months, she had noticed increasing weakness in her hands and legs.

Examination showed this patient to be the most severely affected of the series. Movements of the iliopsoas and glutei were not possible against gravity. There was, in addition, weakness of the triceps and the supraspinatus, the infraspinatus and the deltoid groups. The quadriceps were also severely affected but could maintain the limbs against gravity. This patient also showed moderate weakness of the extensors and flexors of the wrists.

Biopsy revealed a great amount of fat and fibrous tissue replacement of muscle fibres. Some normal muscle fibres, however, were still present, and they were of normal calibre. One necrotic area was found with an apparent phagocytic response.

In spite of the far advanced state, this patient responded well to cortisone, though not to the same degree as did the others. She could not rise from a chair, even with the aid of her arms, before therapy, but she did so readily at the close of treatment. Other muscle power improved correspondingly. Moderate contractures of the calf muscles and achilles tendons softened somewhat during treatment. She is now on maintenance therapy.
Case 5 (L. B., aged 56).-This patient had noticed weakness of the shoulder and hip muscles for two years. She stated that she could not elevate her arms above the horizontal, could not climb stairs or rise from low chairs, and had been subject to frequent falls.

Examination revealed the characteristic weakness of the proximal muscle groups, but there was also some shoulder arthropathy with limitation of abduction of the arms to $90^{\circ}$. Primary weakness was in the iliopsoas, the glutei, and the quadriceps. There was minimal involvement of the deltoids and supraspinati.

This patient responded rapidly to cortisone therapy. Interestingly enough, she showed the most rapid decline of all patients when on cholesterol control, and was as bad as ever by the seventh control day. She could be made to improve and relapse by alternating the regime of cortisone or cholesterol without her knowledge.

\section{Discussion}

Among the myopathies of late onset there is a group that can be distinguished both clinically and pathologically from the others. This condition is quite common. It may be wrongly laid to the general enfeeblement of age, especially since muscular wasting is seldom evident. It is characterized by weakness of the proximal muscles of the hip and shoulder girdles. It is manifested by difficulty in rising from recumbent or sitting positions or in climbing stairs and by a tendency to un predictable falls.

The pathological process is different from that of juvenile muscular dystrophy. In the juvenile form the muscular dystrophy is that of degeneration by? reduction in size of isolated muscle fibres surrounded by essentially normal fibres. The myopathy we are discussing has, in addition, a degeneration of isolated fibres which are undergoing necrotic changes. There is a dissolution of the sarcolemmal sheath, aggregation of the sarcoplasm, loss of cross striation, and an apparent clustering of the sarcolemmal nuclei. This apparently calls forth, in addition, an apparent phagocytic response. There is minimal interstitial infiltration of wandering histiocytes. This is similar to the picture described by Pappenheimer and others in vitamin E-deficient animals which could be protected by tocopherols. Some of our cases were aided by wheat germ oil. Because of this fact, and also because such dystrophic processes occur at the time of endocrine disturbance (menopause), these patients were placed upon cortisone, with the dramatic results noted above. The best guide to diagnosis appears to be in muscle biopsy, but all patients showing this clinical picture should be given a trial of cortisone therapy.

The term "menopausal muscular dystrophy" is debatable, since there is no proof of gonadal dysfunction. It is, however, the best term we can think of at the moment. 


\section{Summary}

A myopathy affecting proximal girdle muscles (hips and shoulders) can be distinguished from other myopathies of late onset by a typical pathological picture. This condition occurs in the post-climacteric period. In this series it was found predominantly in the female.

The necessity of biopsy for diagnosis is emphasized.

Some of these patients have been treated over long periods of time with wheat germ oil, with temporary relief for periods up to three years.

The similarity of the pathological changes to those described in vitamin E-deficient animals is discussed.

These patients have responded well to administration of cortisone acetate. Maintenance dosage is necessary.
We are grateful to Miss Mona Landy and Miss Doris Brophy for skilled technical assistance. We thank Mr. Charles Hodge for photographic help.

\section{REFERENCES}

Barnes, S. (1932). Brain, 55, 1.

Bramwell, E. (1922). Proc. roy. Soc. Med., 16, Sect. Neurol., p. 1.

Hoefer, P., and Glaser, G. H. (1950). J. Amer. med. Ass., 143, 620.

Malamud, N., Nelson, M. M., and Evans, H. M. (1949). Ann. N.Y. Acad. Sci., 52, Art. 3, 135-138.

Medical Research Council (1942). "Aids to the Investigation of Peripheral Nerve Injuries." War Memo. No. 7. H.M.S.O.

Nevin, S. (1936). Quart. J. Med., 5 N.S.. 51.

Pappenheimer, A. M. (1943a). Res. Publ. Ass. neıv. ment. Dis., 22, 85.

(1943b). Physiol. Rev., 23, 37.

Shy, G. M., Brendler, S., Rabinovitch, R., and McEachern, D. (1950). J. Amer. med. Ass., 144, 1353. 